\title{
Revisão sistemática de estudos sobre registros de violência contra crianças e adolescentes no Brasil
}

\author{
Systematic review of studies on reports of violence \\ against children and adolescents in Brazil
}

Davi Manzini Macedo ${ }^{1}$

Laura Nichele Foschiera ${ }^{2}$

Thays Carolyna Pires Mazzini Bordini ${ }^{2}$

Luísa Fernanda Habigzang ${ }^{2}$

Silvia Helena Koller ${ }^{1}$

${ }^{1}$ Instituto de Psicologia, Universidade Federal do Rio Grande do Sul. Av. Paulo

Gama 110, Farroupilha. 90040-060 Porto Alegre RS Brasil.

davimanzini@gmail.com

${ }^{2}$ Programa de Pós-

Graduação em Psicologia,

Pontifícia Universidade

Católica do Rio Grande do

Sul. Porto Alegre RS Brasil.
Abstract This systematic review investigates how violence against children and adolescents data have been accessed, analyzed and discussed in Health scientific literature in Brazil. A sample of 50 articles, based in violence cases registered in the period from 1990 to 2015, was selected throughout the bases SciELO, PePSIC, Lilacs, Web of Science e Scopus. The analysis of frequency distribution pointed out that the major document typology consulted was the violence notification to the Guardianship Council. Characteristics of victims and of the violence situation were the most frequently analyzed. The data set permitted infer about the evolution of the violence epidemiological vigilance in the period considered. The Thematic Analysis showed that data was discussed in relation to the different contextual levels in which violence is manifested. The high absence of the information registered and the inexistence of psychosocial variables precedents to the violent situations interfered in the appliance of recent theoretical models.

Key words Mandatory notification, Violence, Child, Adolescent, Brazil
Resumo A presente revisão sistemática investiga como registros de violência contra crianças e adolescentes, no Brasil, são acessados, analisadose discutidos em artigos científicos da área da saúde. Foram selecionados 50 artigos das bases SciELO, PePSIC, LILACS, Web of Science e Scopus baseados em dados do período de 1990 a 2015. A análise de distribuição de frequência caracterizou os registros utilizados nas publicações. Identificou-se que o documento mais utilizado foi a notificação de violência ao Conselho Tutelar. As características das vítimas e das situações de violência foram as mais frequentemente analisadas. Os dados observados informaram sobre a evolução das politicas de vigilância epidemiológica da violência no país. Por meio da Análise Temática, constatou-se que os dados foram discutidos e relacionados aos diferentes niveis contextuais em que se manifesta a violência. Os resultados mostram elevados indices de informações ausentes entre os registros, bem como desconhecimento de variáveis psicossociais anteriores às situações de violência que interferem na testagem dos dados a partir de modelos teóricos atuais.

Palavras-chave Notificação de abuso, Violência, Criança, Adolescente, Brasil 


\section{Introdução}

O objetivo desta revisão sistemática foi identificar e avaliar artigos científicos da área da Saúde baseados em registros de violência contra crianças e adolescentes no contexto brasileiro, publicados no período entre 1990, marco da Lei no ${ }^{\circ} .8 .069$, e abril de 2015. Os objetivos deste estudo foram: 1 ) caracterizar as publicações baseadas em registros de violência na infância e adolescência e as amostras investigadas por esses estudos e 2) identificar temas principais na seção discussão de resultados dessas publicações

A violência contra crianças e adolescentes é reconhecida internacionalmente como um grave problema de Saúde Pública e de Direitos Humanos. Consiste em toda forma de maus tratos que ocorra em uma relação de responsabilidade ou poder e que resulte em dano à dignidade, saúde e desenvolvimento de crianças e adolescentes ${ }^{1}$. As implicações da violência podem estender-se desde gastos em programas de proteção e sistema prisional até prejuízos nas esferas da saúde e qualidade de vida, além de óbito. Apenas no ano de 2012, foram identificadas 95.000 vítimas de homicídio com idades entre zero e 19 anos, com o maior número de mortes concentrado na América Latina e Caribe. As formas mais frequentes de violência contra crianças e adolescentes são a negligência e as violências física, psicológica e sexual ${ }^{2}$.

A partir da promulgação da Lei n. 8.069, o Estatuto da Criança e do Adolescente - ECA, a legislação brasileira reconhece a infância e a adolescência como fases de desenvolvimento peculiares e estabelece a necessidade de proteção integral à criança e ao adolescente a fim de lhes garantir o desenvolvimento em condições de liberdade e dignidade. Notificações de suspeitas ou confirmações da violência foram estabelecidas como compulsórias e devem ser reportadas ao Conselho Tutelar, conforme o Artigo no13 do ECA. Esse é o órgão de referência para acolhimento dos casos e encaminhamento das vítimas e familiares aos serviços de atendimento. A notificação também pode ser realizada junto a outras instituições, que devem atuar articuladas ao Conselho Tutelar, como as Delegacias de Proteção da Criança e do Adolescente e o Ministério Público ${ }^{3}$.

A legislação também estabelece como compulsória a notificação da violência no âmbito da Saúde, com a finalidade de monitorização de sua ocorrência por meio de ações articuladas e sistematizadas ${ }^{4}$. O Sistema de Vigilância de Violências e Acidentes (VIVA), criado em 2006, foi a primeira iniciativa de sistematização nacional de dados sobre violência. A partir de 2009, o componente de vigilância contínua de violência doméstica, sexual, e/ou outras violências interpessoais e autoprovocadas foi integrado ao Sistema de Informação de Agravos de Notificação, um sistema de dados de endemias e agravos já consolidado em nível nacional ${ }^{5}$.

Crianças e adolescentes vítimas de violência realizam um percurso complexo e não sistematizado dentro das instituições e órgãos dos setores Educação, Assistência Social, Saúde, Segurança Pública e Justiça no Brasil. Tal percurso tem como objetivo atender às necessidades de tratamento das consequências da violência, de responsabilização dos agressores e de prevenção de novas ocorrências. Conselhos Tutelares, Centros de Referência de Assistência Social, Varas de Infância e Juventude, Institutos Médico Legais, bem como unidades de saúde, hospitais e programas de atendimento às vítimas, são órgãos componentes da rede de proteção.

O fluxo de atendimento em diversos níveis promove a criação de registros sobre os casos em todas estas instituições. Estes registros podem apresentar diferentes formatos, a depender da função do serviço prestado e dos avanços das políticas que estabelecem a compulsoriedade da notificação. A integração de dados referentes à violência permite estimar os investimentos necessários ao aperfeiçoamento da rede de proteção ${ }^{7}$. A compreensão desses dados pode ser beneficiada pela aplicação de referenciais teóricos que considerem os diferentes níveis contextuais envolvidos na gênese da violência, bem como as possíveis intersecções entre eles ${ }^{8}$. Em programas preventivos desenvolvidos pela World Health Organization (WHO), a Abordagem Bioecológica do Desenvolvimento Humano $(\mathrm{ABDH})^{9,10}$ é apontada como referência para identificação dos diferentes níveis onde a violência ocorre e como interagem os fatores de risco presentes em cada um desses contextos ${ }^{11}$.

A aplicação da $\mathrm{ABDH}$ propõe a integração de características das crianças (e.g., período desenvolvimental, sexo, temperamento), dos cuidadores (e.g., vínculo com a vítima, nível de estresse, história prévia de violência) e do ambiente familiar (e.g., recursos econômicos disponíveis, estrutura física ambiental) para compreensão da etiologia da violência. Consideram, ainda, aspectos do contexto social mais amplo (e.g., configuração da rede de apoio, acesso a bens e serviços) e de concepções e práticas socioculturais que corroboram para a tolerância à violência (e.g., violência como prática educativa) $)^{8,12,13}$. 
Ao enfatizar os níveis ecológicos nos quais a violência se manifesta, a $\mathrm{ABDH}$ permite distinguir fatores de risco para ocorrência da violência e identificar fatores protetivos. Dessa forma, pode auxiliar no desenvolvimento de intervenções a nível de saúde pública ao identificar, de modo mais preciso, onde as estratégias planejadas devem ser aplicadas. Permite também a avaliação de políticas públicas, ao apontar os mecanismos que apresentam resultados e em qual nível contextual isso acontece ${ }^{11}$.

$\mathrm{O}$ objetivo da presente revisão sistemática foi verificar como dados de situações de violência contra crianças e adolescentes, no Brasil, têm sido acessados e analisados no âmbito científico da saúde. Visa, ainda, identificar eixos temáticos na seção de discussão de resultados dos artigos e verificar se as dimensões do modelo bioecológico tem sido consideradas para discussão da violência contra crianças e adolescentes no contexto brasileiro. Esse estudo intenta demonstrar quais fontes tem servido à análise epidemiológica da violência contra crianças e adolescentes no Brasil. A análise de eixos temáticos comuns às discussões empreendidas, visa a verificação de quais níveis de compreensão da violência contra crianças e adolescentes tem sido possibilitada pela análise dos registros consultados.

\section{Método}

\section{Procedimentos de coleta de dados}

Foram consultadas, por dois juízes independentes, cinco bases de dados indexadas na área das Ciências da Saúde: SciELO, PePSIC, LILACS, Web of Science e Scopus. Os descritores foram formulados em língua portuguesa e inglesa e submetidos à consulta nos Descritores em Ciências da Saúde (DeCS) da Biblioteca Virtual de Saúde. Os strings resultantes foram: (Violência $O R$ Maus Tratos OR Abuso) AND (Infância OR Adolescência $O R$ Crianças $O R$ Adolescentes $O R$ Jovens $O R$ Juventude) AND (Notificação $O R$ Denúncia $O R$ Epidemiologia $O R$ Perfil $O R$ Incidência $O R$ Prevalência) e os equivalentes em inglês (Violence OR Maltreatment OR Abuse) AND (Childhood OR Adolescence OR Children OR Adolescents OR Young OR Youth) AND (Notification OR Denunciation OR Epidemiology OR Profile OR Incidence OR Prevalence).

Para as bases Web of Science e Scopus acrescentaram-se os descritores Brazil e Brazilian para que fossem selecionados artigos baseados em da- dos do Brasil. As buscas em todas as plataformas consultadas dataram de 16 a 30 de abril de 2015. Os resultados exibidos corresponderam a 2804 resumos. A base PepSic forneceu 39 resultados, a Scielo resultou em 390 artigos e as bases LILACS, Web of Science e Scopus resultaram em 1846, 210 e 316 artigos, respectivamente.

Os resultados das buscas foram exportados das bases de dados e importados pelo software State of Art Through Sistematic Review (Start). Por meio do programa, foram identificadas e excluídas 826 repetições entre as bases de dados. $\mathrm{O}$ processo de seleção dos 1978 resumos também foi realizado por dois juízes independentes. Procedeu-se a leitura dos títulos e, caso identificada a temática da violência, os resumos foram analisados a partir de cinco critérios de inclusão (C.I.): (1) Os artigos devem ser baseados em registros de situações de violência no Brasil; (2) Os dados devem corresponder a situações de violência contra crianças e adolescentes; (3) A função da análise dos dados deve ser a caracterização epidemiológica; (4) Os artigos devem ser baseados em situações de violência identificadas entre 1990 e 2015; (5) A versão completa das publicações deve estar disponível.

O equivalente a 77 resumos foi considerado elegível. Buscou-se a versão completa dos artigos e procedeu-se a releitura dos resumos e, caso necessário, da seção método a fim de verificar se atendiam aos critérios de exclusão (C. E.): (1) artigos duplicados; (2) revisões sistemáticas e meta-análises; (3) teses e dissertações; (4) artigos baseados em dados que envolviam comorbidades; (5) artigos não baseados em registros de informações e, (6) presença de dados de grupos etários posteriores à adolescência. Nenhuma meta-análise, revisão sistemática ou artigos publicados em idioma diverso do português, inglês ou espanhol constou nos resultados. Foram acrescentados três artigos a partir de busca nas referências dos artigos encontrados. A amostra final totalizou 50 artigos. O processo de seleção dos artigos é ilustrado na Figura 1 e a lista completa das referências selecionadas foi disponibilizada em arquivo suplementar.

As categorias de análise utilizadas foram: (1.1) caracterização do período das publicações analisadas; (1.2) caracterização da área específica da Saúde à qual pertencem às publicações; (1.3) delimitação do período de registro das situações de violência; (1.4) caracterização da localidade de origem dos registros por região do país; (1.5) identificação das instituições de origem dos registros; (1.6) identificação das unidades de regis- 


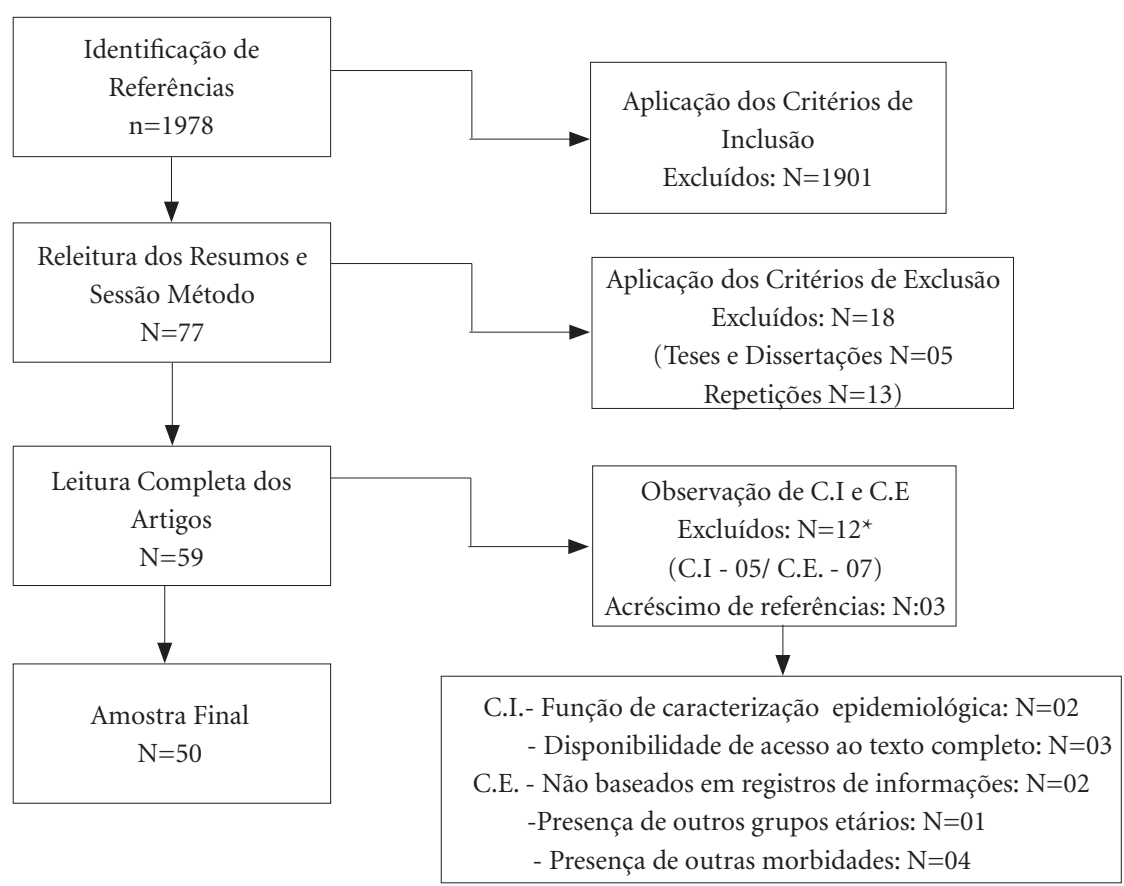

Figura 1. Estratégias de seleção da amostra

tro utilizadas na amostra (e.g. prontuários, processos judiciais, sistemas informatizados); (1.7) caracterização do tamanho amostral utilizado para análise; (1.8) identificação das principais variáveis selecionadas para análise (e.g., tipologia de violência, sexo das vítimas, vínculo entre vítima e agressor, consequências da violência); (1.9) verificação da avaliação realizada pelos autores em relação à qualidade dos dados utilizados; (1.10) identificação das análises estatísticas empreendidas (descritivas $x$ inferenciais) e 2.0) identificação de eixos temáticos na discussão dos dados analisados

Os dados dos artigos analisados foram extraídos com o auxílio do programa Start. Foi utilizado um formulário de extração de dados elaborado de acordo com os objetivos de pesquisa e testado previamente com cinco artigos da amostra. Após a fase de extração, os dados foram tabulados pelo programa Start para o formato Microsoft Word Excel 2007 e, posteriormente, para o Statistical Package for the Social Sciences (SPSS) versão 21.0.

\section{Procedimentos de análises de dados}

Análises estatísticas descritivas foram realizadas no SPSS com vias à obtenção de distribuições de frequências simples das variáveis analisadas. A análise da seção de discussão dos artigos foi realizada por meio da Análise Temática, método flexível e sistematizado para a criação de eixos (temas) de análise coerentes e consistentes com os objetivos de pesquisa. Buscou-se investigar quais os principais eixos temáticos que permeavam a discussão sobre os dados de violência ana$\operatorname{lisados}^{14}$.

\section{Resultados}

\section{Caracterização das publicações e das amostras utilizadas}

Os artigos selecionados eram referentes às áreas de Saúde Pública/Coletiva (28\%), Enfermagem (22\%), Psicologia (18\%), Medicina/ Pediatria (9\%), Odontopediatria (8\%) e outros (6\%). O maior percentual de publicações ocorreu entre 2011 e 2015 (42\%) e no intervalo entre os anos de 2006 e 2010 (40\%). Quanto ao período de registro das situações de violência, o per- 
centual de $46 \%$ dos artigos foi baseado em casos registrados entre 2006 e 2010. O número de casos analisados nos artigos variou entre 14 e 17.900 . Em 12 artigos (24\%), as amostras variaram entre 14 e 100 casos, em 24 artigos (48\%) as amostras variaram entre 100 e 1000 casos, em 11 artigos $(22 \%)$ as amostras variaram entre 1000 e 10.000 casos e em três artigos (6\%) as amostras foram superiores a 10.000 casos. Quanto à localidade do registro, destaca-se o percentual de $34 \%$ da região Sul, enquanto $30 \%$ foram provenientes da região Nordeste, $26 \%$ da região Sudeste, $4 \%$ da região Norte e $2 \%$ da região Centro-Oeste. $\mathrm{O}$ equivalente a $4 \%$ dos artigos analisou registros provenientes de todo o território nacional.

A natureza dos registros das situações de violência foi analisada a partir das seguintes categorias: Processos judiciais, Prontuários de atendimento, Notificações, Exames periciais físicos, Ocorrências/processos policiais e Outros. Destaca-se que 6\% dos artigos utilizaram mais de uma categoria de registro de dados. Aquelas com maior prevalência foram Notificações de violência (56,6\%) e Prontuários de atendimento (11,3\%). As notificações de violência foram provenientes, sobretudo, dos Conselhos Tutelares (50\%), mas também de programas de atendimento especializado (30\%) e dos sistemas de informação VIVA e SINAN (20\%). Os prontuários de atendimento foram originados de centros especializados no atendimento infanto-juvenil (50\%) e de violência sexual (16,6\%), assim como de Centros de Referência Especializado de Assistência Social (CREAS; $16,6 \%$ ) e de programas de atendimento específico às crianças vitimizadas (16,6\%).

Quanto às outras categorias de natureza dos registros, destacam-se os exames periciais físicos $(9,4 \%)$, provenientes em sua totalidade de Institutos Médico Legais, assim como as ocorrências/ processos policiais $(7,5 \%)$, cuja proveniência foi exclusivamente de delegacias. Os dados de processos judiciais representam 5,7\% dos registros e foram provenientes de Juizados de Infância e Juventude $(66,6 \%)$ e do Ministério Público $(33,3 \%)$. A categoria Outros $(9,4 \%)$ correspondeu a prontuários de atendimento hospitalar (40\%), laudos psicológicos e psiquiátricos (20\%), registros de mortalidade (20\%) e entrevistas com vítimas e acompanhantes com a finalidade de complementar informações de outras formas de registro (20\%).

As variáveis com maior prevalência de análise foram as referentes às características das vítimas (e.g. sexo, idade, cor da pele, escolaridade) em 96\% dos artigos, e da situação de violência em si (e.g., local de ocorrência, tipologia de violência, meios de agressão) em 90\% das publicações. As variáveis referentes às características dos agressores (e.g. sexo, faixa etária, vínculo com a vítima) e às características do atendimento (e.g. encaminhamentos, dados de acompanhamento) foram analisadas em $76 \%$ e $46 \%$ dos artigos, respectivamente. As consequências da violência (e.g. presença de lesões, sequelas) foram referidas por $34 \%$ dos artigos.

Em 72\% dos artigos analisados observou-se a indicação de ausência de informações entre as variáveis investigadas. Apenas 20\% dos artigos reportou a utilização de testes estatísticos inferenciais para o tratamento dos dados. Os demais apresentaram análises estatísticas descritivas. $\mathrm{Na}$ Tabela 1 constam informações sobre a distribuição de frequência da localidade dos registros, das unidades de registro consultadas e das variáveis selecionadas para análise.

\section{Eixos temáticos identificados na seção de discussão de resultados das publicações}

Foram construídos dez eixos temáticos a partir dos temas principais identificados na seção de discussão de dados das publicações (Tabela 2). Destaca-se o eixo Aspectos Socioculturais, Políticos e Econômicos, presente em 21,9\% da amostra. Este eixo compreendeu discursos referentes ao papel de fatores como desemprego, baixa condições de acesso a bens de consumo, serviços, informação e direitos básicos de cidadania como fatores que contribuem para a consolidação de situação de violência em âmbito intrafamiliar. Foi também destacada a influência do gênero da vítima na forma de manifestação dos atos praticados a partir do processo de socialização diferenciado e pelos comportamentos socialmente valorizados para cada gênero (e.g., maior liberdade para os meninos e maior vigilância sobre as meninas, armas como simbologia da poder masculino).

Outro eixo recorrente foi Atuação da Rede de Proteção (16,4\%), que compreendeu discussões pautadas na avaliação do exercício dos profissionais envolvidos na identificação e manejo dos casos. Estão presentes discussões referentes ao preparo dos conselheiros tutelares e de profissionais da Saúde e Educação no que tange à identificação, acolhimento, encaminhamento e notificação. O eixo Padrões de Ocorrência da Violência $(16,4 \%)$ incluiu extratos baseados em padrões comuns às situações de violência contra crianças e adolescentes (e.g., preponderância do ambiente familiar como local de ocorrência, existência 


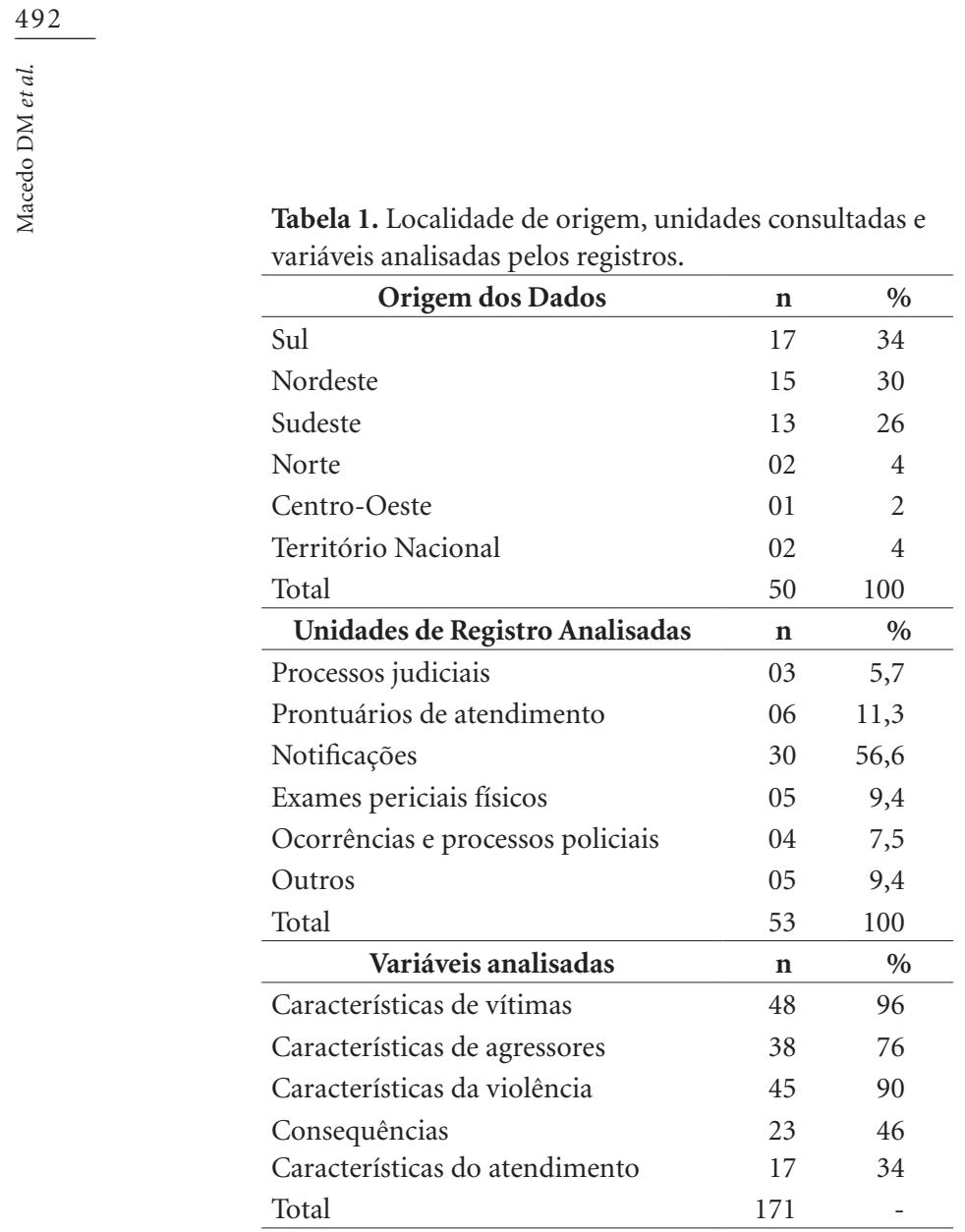

Tabela 2. Eixos temáticos identificados na discussão dos registros de violência.

\begin{tabular}{lcc}
\hline \multicolumn{1}{c}{ Eixos Temáticos } & n & \% \\
\hline Aspectos socioculturais, políticos e & 16 & 21,9 \\
econômicos & & \\
Atuação da rede de proteção & 12 & 16,4 \\
Padrões de ocorrência da violência & 12 & 16,4 \\
Comparação das distribuições de frequência & 10 & 13,7 \\
Consequências da violência & 07 & 9,6 \\
Aspectos desenvolvimentais das vítimas & 04 & 5,5 \\
Avaliação de políticas de vigilância & 03 & 4,1 \\
epidemiológica & & \\
Características do(a) agressor(a) & 03 & 4,1 \\
Fatores de risco e proteção & 03 & 4,1 \\
Qualidade da informação registrada & 03 & 4,1 \\
Total & 73 & 100 \\
\hline
\end{tabular}

de vínculo de confiança entre vítima e agressor, ocorrência de mais de um tipo de violência, utilização de ameaças e agressões para manter o silêncio das vítimas).

O eixo "Comparação das Distribuições de Frequência" (13,7\%) disse respeito às discussões

limitadas ou focadas à comparação dos resultados com taxas de incidência de outros estudos, sem a presença de argumentos para explicação dos resultados. Já o eixo "Consequências da Violência" $(9,6 \%)$ foi referente ao apontamento de sintomas, predominantemente em níveis físico e emocional, comumente associados às tipologias de violência investigadas.

Entre os eixos com menor frequência, "Aspectos Desenvolvimentais das Vítimas" (5,5\%) tratou da discussão de características intrínsecas à infância e adolescência que podem influenciar no modo de manifestação da violência, como vulnerabilidade, dependência de cuidados externos e imaturidade biopsicossocial. O eixo "Avaliação das Políticas de Vigilância Epidemiológica" $(4,1 \%)$ correspondeu à discussão a respeito das diferentes fases da implementação de estratégias de vigilância dos casos de violência no país.

Em "Fatores de Risco e Proteção" $(4,1 \%)$ estão presentes discussões que compreenderam aspectos de risco e de proteção para a ocorrência e reincidência das situações de violência, como características das vítimas, da dinâmica familiar e da atuação da rede de proteção. O eixo temático "Qualidade das Informações Registradas" (4,1\%) envolveu discussões centradas nas características dos registros de violência e nos fatores que podem comprometer a quantidade e validade dos dados disponíveis. Por fim, o eixo "Perfil do Agressor" $(4,1 \%)$ se referiu às discussões pautadas em características do agressor e sua variação em função da tipologia de violência (e.g., figuras parentais geralmente associadas aos casos de negligência).

\section{Discussão}

\section{Caracterização das publicações e das amostras utilizadas}

A identificação de publicações em diferentes áreas da Saúde reflete a multidimensionalidade da violência ${ }^{2}$ e pode representar a necessidade de integração de diferentes saberes profissionais para a identificação e manejo dos casos. Já o período das publicações analisadas, mais frequentes a partir de 2006, e o período de ocorrência das situações analisadas, concentradas, sobretudo, entre os anos de 2006 e 2010, sugerem a lenta implementação da vigilância epidemiológica da violência contra a infância e adolescência no Brasil. A menor frequência de publicações no período anterior considerado a luz do ECA, que preco- 
niza, desde 1990, a prevenção da violência neste período desenvolvimental de vulnerabilidade ${ }^{3}$, indica que a consolidação das análises desses registros ocorreu de maneira tardia.

Um percentual considerável de artigos apresentou amostra inferior a 100 casos, enquanto apenas $28 \%$ basearam-se em amostras superiores a 1.000 casos, o que indica que no período analisado foi elevada a análise de dados circunscritos a amostras limitadas do ponto de vista estatístico. A maioria das análises estatísticas empreendidas foi a análise de distribuição de frequências. Somado à incompletude dos dados, esse aspecto limita as associações que podem ser realizadas a partir dos dados e prejudica a identificação de fatores de risco e proteção envolvidos. $\mathrm{O}$ aumento recente do número de casos registrados, contudo, é um indicativo do avanço da análise epidemiológica da violência, embora sua recenticidade corrobora a perspectiva de que esta evolução ocorre de modo lento ${ }^{15}$.

Os registros de informações foram provenientes das regiões Sul, Nordeste e Sudeste, majoritariamente. A baixa prevalência de estudos provenientes da região Norte e Centro-Oeste está em consonância com investigação baseada em notificações de violência contra crianças junto ao SINAN no ano de 2010 em todo o território nacional. As análises evidenciaram que os menores percentuais de notificação correspondiam aos estados de Rondônia, Amapá, Rio Grande do Norte e Tocantins ${ }^{15}$. A comparação entre municípios e federações, contudo, é dificultada em função dos diferentes estágios de implementação das políticas de vigilância epidemiológica. Ao invés de indicar a incidência da violência em uma respectiva localidade, esta comparação pode indicar diferenças no comprometimento dos gestores e das equipes técnicas para a implementação e melhoria da qualidade do registro e análise de dados de violência ${ }^{16}$.

O destaque de dados provenientes dos Conselhos Tutelares indica sua posição como o principal órgão responsável pelo acolhimento de crianças e adolescentes em situação de violação de direitos ${ }^{3}$. Apesar das dificuldades decorrentes da falta de recursos e de estrutura física ${ }^{7}$, o conjunto de dados analisado indica que essas instituições têm sido minimamente capazes de fornecer informações relacionadas às situações de violência atendidas. Destaca-se que a compilação de tais informações por estas instituições é essencial enquanto outras estratégias de análise epidemiológica dos aspectos envolvidos na etiologia da violência são implementados e aprimorados.
A categoria de registro com maior destaque nas publicações analisadas foi a de notificação da violência. Foram originadas, sobretudo, de Conselhos Tutelares, cuja finalidade primeira é interrupção da violência e encaminhamento dos envolvidos para rede de atendimento ${ }^{3}$. Também identificaram-se notificações próprias de programas de atendimentos em nível municipal e estadual, que podem ser consideradas comprometimento de gestores locais com o registro e a análise de informações. Podem ser consideradas estratégias particulares para suprir a ausência de sistemas unificados.

Ainda foram constatadas notificações do sistema de informação em Saúde, cuja finalidade é a vigilância epidemiológica. Os sistemas informatizados representam a iniciativa do Ministério da Saúde, que desde 2001 visa a implantação de uma ficha padronizada de notificação em todas as unidades de saúde pública do país a fim de caracterizar a violência, o perfil dos envolvidos e os encaminhamentos realizados ${ }^{17}$. O resultado de tal sistematização pode ser observado nas publicações analisadas. Os artigos com amostra igual ou superior a 10.000 casos foram baseados em dados registrados a partir de 2006 e, sobretudo (66,6\%) em dados do SINAN. Essa base foi a única fonte, destaca-se, capaz de fornecer dados que contemplassem todo o território nacional. Esta constatação evidencia o potencial destes sistemas para gerar grandes volumes de dados e subsidiar análises sofisticadas para elaboração de um perfil fidedigno de vítimas e suspeitos de agressão.

Apesar dos progressos observados, a subnotificação das situações de violência ainda é uma realidade. Além de estar associada a fatores presentes na dinâmica da violência, a subnotificação pode ser agravada por aspectos externos, relacionados à administração governamental e à gestão de políticas públicas ${ }^{18}$. Outros fatores intervenientes podem ser a falta de capacitação profissional para identificação de casos, ausência de conhecimento sobre a compulsoriedade da notificação e preceitos do ECA $^{19}$, ausência de articulação entre os serviços da rede para adoção das medidas de proteção, medo de exposição por parte dos profissionais e banalização da violên$\mathrm{cia}^{20}$.

Além da subnotificação, o apontamento de ausência de informações entre as variáveis foco de investigação foi considerável. Algumas das informações, sobretudo as referentes às consequências e aos encaminhamentos realizados, estavam incompletas em grande parte dos registros analisados. Este fato é apontado na maioria dos 
artigos da amostra, inclusive nos mais recentes. A ausência de informações sobre as consequências da violência pode ser explicada pela inadequação das condições oferecidas no momento da coleta. Tal identificação requer o diagnóstico clínico e o acompanhamento das vítimas por profissionais capacitados, uma vez que os critérios para muitos transtornos psicológicos e psiquiátricos dependem da manutenção dos sintomas por períodos determinados ${ }^{21}$. A coleta de dados dessa natureza requer, portanto, a reorganização dos serviços implicados para a identificação e monitoramento dos sintomas reportados.

A ausência de dados sobre os encaminhamentos, por sua vez, pode refletir a ausência de um fluxo padronizado para atuação da rede, o que pode centrar na avaliação subjetiva do profissional as decisões sobre quais seriam as necessidades de atendimento da criança ou adolescente ${ }^{22}$. Esta falha na compilação de dados corrobora a perspectiva de que o percurso realizado pelas crianças e adolescentes vítimas nos serviços da rede de proteção carece de padronização ${ }^{7}$. A inexistência de um fluxo determinado a priori compromete também a análise do atendimento realizado e minora a avaliação e reelaboração das estratégias de intervenção adotadas.

Eixos Temáticos Identificados na Seção de Discussão de Resultados das Publicações

Os eixos temáticos construídos a partir da seção de discussão dos resultados nas publicações evidenciam a complexidade e os diferentes fatores que permeiam o fenômeno da violência. Destaca-se que a maioria dos artigos buscou discutir os dados a luz de múltiplos fatores e as categorias temáticas são ilustrativas dos principais temas abordados. Constatou-se que os temas identificados consideram fatores dos diferentes níveis contextuais envolvidos na gênese e perpetuação da violência ${ }^{8,12,13}$.

Os eixos temáticos Aspectos Desenvolvimentais das Vítimas e Características do(a) Agressor(a) foram identificadas a partir de discussões a respeito de aspectos que interagem nas relações imediatas dos envolvidos, como o ambiente intrafamiliar. Os eixos Atuação da Rede de Proteção, Avaliação das Políticas de Vigilância Epidemiológica e Qualidade da Informação Registrada referem-se, por sua vez, a aspectos do contexto social ampliado, visto que referem-se à organização dos serviços de atendimento oferecidos às vítimas, suspeitas de agressão e seus familiares, bem como à avaliação das estratégias preventivas, nomeadamente o registro das informações e a análise epidemiológica.
O eixo Aspectos Socioculturais, Políticos e Econômicos contempla as concepções em nível macro que fomentam e/ou toleram a prática da violência contra a infância e a adolescência. Considera-se que esta divisão em níveis é didática e pressupõe-se sua interseccionalidade, conforme a ABDH, na qual esses modelos são basea$\operatorname{dos}^{9,10}$. Tal intersecção é evidenciada pelos eixos Padrões de Ocorrência da Violência, Fatores de Risco e Proteção e Consequências da Violência, que compilam aspectos dos diferentes níveis para identificação e classificação de aspectos recorrentes da violência e de suas consequências.

Embora a discussão empreendida evidencie que as variáveis coletadas pelos registros permite a discussão dos fatores e contextos envolvidos na gênese da violência, essa constatação aponta limitações. Os registros utilizados, mesmo os dos sistemas informatizados, não fornecem informações a respeito de diversas variáveis psicossociais apontadas pela $\mathrm{ABDH}$ como atuantes na gênese da violência. Foi também pouco frequente a discussão de fatores de risco e proteção, cuja aplicabilidade é apontada por organizações de referência ${ }^{11}$. Variáveis não investigadas que podem contribuir para compreender especificidades da violência no país são: nível socioeconômico familiar, ocupação dos cuidadores, número de habitantes da residência, período desenvolvimental dos pais, nível de estresse parental e histórico de vitimização dos cuidadores ${ }^{8,12,13}$.

Sugere-se que a coleta de variáveis que permitam a testagem da adequação dos modelos propostos à realidade brasileira exigiria a articulação dos serviços envolvidos no manejo de situações de violência na infância e na adolescência. A atuação conjunta dos âmbitos Saúde, Educação, Assistência Social, Segurança Pública e Justiça para coletar informações pertinentes às suas áreas de atuação e sistematizá-las de modo integrado, permitiria análises multifatoriais, conforme exige o fenômeno em questão. Empecilhos para alcançar esse panorama são a inexistência de um fluxo protocolar para o atendimento das vítimas e demais envolvidos, bem como os estágios iniciais de consolidação de um sistema unificado de vigilância epidemiológica da violência no país.

Para fins de conclusão, aponta-se que a utilização de diferentes fontes de registros para análise das situações de violência representa a diversidade de instituições e serviços envolvidos no processo de atendimento à criança e ao adolescente vítimas. Observou-se a preponderância de dados advindos dos Conselhos Tutelares, em função de sua centralidade no acolhimento e en- 
caminhamento dos casos aos outros órgãos da rede. A notificação de violência, seja as registradas pelos Conselhos Tutelares ou pelos sistemas informatizados, foram as fontes de dados mais relevantes. Observou-se aumento na qualidade das descrições a partir das situações registradas pelos sistemas VIVA e SINAN, o que pode ser um indicativo dos resultados da padronização da coleta e registro dos dados. Ainda assim, observamse pontos a serem trabalhados para a melhoria do atendimento e registros de informações pertinentes aos casos, como o preenchimento completo das informações solicitadas e aperfeiçoamento na coleta e análise de dados nas diferentes regiões do país.
Verificou-se que os eixos temáticos a partir dos quais foram discutidos os dados analisados representam os diferentes níveis contextuais implicados na gênese da violência. Os registros brasileiros, contudo, apresentam limitações com relação às variáveis psicossociais que poderiam auxiliar na verificação da adequabilidade destes modelos à realidade brasileira. Considera-se que alcançar esse objetivo requer a articulação entre profissionais dos diferentes segmentos da rede de proteção e sua capacitação adequada para a compreensão da relevância política de primar pela vigilância epidemiológica da violência.

\section{Colaboradores}

DM Macedo trabalhou no desenho da pesquisa, revisão literária, metodologia, resultados, discussão e revisão final do texto. LN Foschiera e TCPM Bordini trabalharam na revisão literária, metodologia e resultados. LF Habigzang trabalhou no desenho de pesquisa, metodologia e revisão do texto. Por fim, SH Koller trabalhou em metodologia e revisão final do texto. 


\section{Referências}

1. World Health Organization (WHO). Preventing child maltreatment: a guide to taking action and generating evidence. Geneva: WHO; 2006.

2. United Nations Children's Fund (UNICEF). Hidden in plain sight: a statistical analysis of violence against children. New York: UNICEF; 2014.

3. Brasil. Lei No 8.069, de 13 de julho, 1990. Dispõe sobre o Estatuto da Criança e do Adolescente e dá outras providências. Diário Oficial da União 1990; 16 Jul.

4. Brasil. Portaria No. 737 de 16 de maio, 2001. Regulamenta a política nacional de redução da morbimortalidade por acidentes e violências. Diário Oficial da União 2001; 18 maio.

5. Brasil. Ministério da Saúde (MS). Viva: Vigilância de violências e acidentes, 2006 e 2007. Brasília: Secretaria de Vigilância em Saúde; 2009.

6. Eloy CB. A credibilidade do testemunho da criança vítima de abuso sexual no contexto judiciário. Psicol Cien Prof [journal on the internet] 2012 [cited 2015 Aug 12];32(1):[about 16 p.]. Available from: http://www. scielo.br/pdf/pcp/v32n1/v32n1a17.pdf

7. Deslandes S, Mendes CHF, Lima JS, Campos DS. Indicadores das ações municipais para a notificação e o registro de casos de violência intrafamiliar e exploração sexual de crianças e adolescentes. Cad Saúde Pública [journal on the internet] 2011 Jan-Aug [cited 2015 Jul 23];27(8):[about 13 p.]. Available from: http://www. scielo.br/pdf/csp/v27n8/18.pdf

8. Cicchetti D, Valentino K. An ecological-transactional perspective on child maltreatment: failure of the average expectable environment and its influence on child development. In: Cicchetti D, Cohen DJ, editors. Developmental Psychopathology. Risk, disorder and adaptation. New Jersey: John Wileys \& Sons; 2006. p. 129-201.

9. Bronfrenbrenner U. The ecology of human development: experiments by nature and design. Cambridge: Harvard University Press; 1979.

10. Bronfenbrenner U. Ecological models of human development. In: Gauvain M, Cole M, editors. International Encyclopedia of Education. Oxford: Elsevier; 1994. p. 37-43.

11. World Health Organization (WHO). [Internet]. The Violence Prevention Alliance Approach [cited 2016 Nov 25]. Secretariat of the Violence Prevention Alliance. Available from: http://www.who.int/violenceprevention/approach/en/

12. Belsky J. Child maltreatment: an ecological integration. Am Psychol 1980; 35(4):320-335.

13. Belsky J. Etiology of child maltreatment: a developmental-ecological analysis. Psychol Bull 1993; 114(3):413-434

14. Braun V, Clarke V. Using thematic analysis in psychology. Qual Res Psychol 2006; 3(2):77-101.

15. Assis SG, Avanci JQ, Pesce RP, Pires TO, Gomes DL. Notificações de violência doméstica, sexual e outras violências contra crianças no Brasil. Cien Saúde Colet [journal on the internet] 2012 Sept [cited $2015 \mathrm{Ju}$ 24];17(9):[about 13 p.]. Available at: http://www.scielo. br/pdf/csc/v17n9/a12v17n9.pdf
16. Rates SMM, Melo EM, Mascarenhas MDM, Malta DC. Violence against children: an analysis of mandatory reporting of violence, Brazil 2011. Cien Saúde Colet [journal on the internet] 2015 Mar [cited 2015 Jun 14];20(3):[about 12 p.]. Available from: http://www.scielo.br/pdf/csc/v20n3/pt_1413-8123-csc-20-03-00655. pdf

17. Lima JS, Deslandes SF. A notificação compulsória do abuso sexual contra crianças e adolescentes: uma comparação entre os dispositivos americanos e brasileiros. Interface (Botucatu) [journal on the internet] 2011 JulSep [cited 2016 Mar 12];15(38):[about 13 p.]. Available from: http://www.scielo.br/pdf/icse/v15n38/aop4111. pdf

18. Costa MCO, Carvalho RC, Santa Bárbara JFR, Santos CAS, Gomes WA, Sousa HL. O perfil da violência contra crianças e adolescentes, segundo registros de Conselhos Tutelares: vítimas, agressores e manifestações de violência. Cien Saude Colet [journal on the internet] 2007 Sep-Oct [cited 2015 Aug 8];12(5):[about 14 p.]. Available from: http://www.scielo.br/pdf/csc/v12n5/04. pdf

19. Silva MAI, Ferriani MGC. Domestic violence: from the visible to the invisible. Rev Lat-am Enfermagem [journal on the internet] 2007 [cited 2015 Sept 13];15(2):[about 8p.]. Available from: http://www.scielo.br/pdf/rlae/v15n2/pt_v15n2a13.pdf

20. Bazon MR. Violências contra crianças e adolescentes: análise de quatro anos de notificações feitas ao Conselho Tutelar na cidade de Ribeirão Preto, São Paulo, Brasil. Cad Saúde Pública [journal on the internet] 2008 Feb [cited 2015 Oct 2];24(2):[about 10 p.]. Available from: http://www.scielo.br/pdf/csp/v24n2/10.pdf

21. American Psychiatric Association. DSM-5: manual Diagnóstico e Estatístico de Transtornos Mentais. Porto Alegre: Artmed; 2014.

22. Ferriani MGC, Pelegrino FM. A trajetória da violência doméstica no município de Ribeirão Preto. Rev Bras Enferm [journal on the internet] 2004 [cited 2015 Jun 13];57(3):[about 3 p.]. Available from: http://www.scielo.br/pdf/reben/v57n3/a19v57n3.pdf

Artigo apresentado em 13/12/2016

Aprovado em 16/03/2017

Versão final apresentada em 18/03/2017 